\title{
Positive- and Negative-Sequence Control of Grid- Connected Photovoltaic Systems under Unbalanced Voltage Conditions
}

\author{
Mitra Mirhosseini $^{(1)}$, Josep Pou ${ }^{(1),(2)}$, Baburaj Karanayil ${ }^{(1)}$ and Vassilios G. Agelidis ${ }^{(1)}$ \\ (1) Australian Energy Research Institute \& School of Electrical Engineering and Telecommunications \\ The University of New South Wales (UNSW), Sydney, NSW 2052, Australia \\ ${ }^{(2)}$ Terrassa Industrial Electronics Group \& Department of Electronic Engineering \\ Technical University of Catalonia (UPC), Catalonia, Spain \\ Email: m.mirhosseini@student.unsw.edu.au
}

\begin{abstract}
This paper discusses the control of the positive- and negative-sequence components of a large-scale grid-connected photovoltaic system (GCPS) under unbalanced voltage sag conditions in the grid. Some issues regarding stability and dynamic performance of the system occur when applying PI controllers in the current control loops. The reason is the delay that the filtering method imposes when extracting the current/voltage sequences. Because of such a delay, the dynamic response of the system becomes slower compared with the case when no filtering technique is needed. Furthermore, there is a strong restriction on choosing suitable parameters for the current/voltage loop controllers without compromising system stability. All these issues are discussed in this paper on a 1-MVA GCPV system using MATLAB/Simulink software.
\end{abstract}

Index Terms - Photovoltaic system, Power system faults, Power system dynamics, Moving average filter.

\section{INTRODUCTION}

Due to the increasing demand of grid-connected photovoltaic systems (GCPSs), some new issues appear [1], which must be taken into consideration for reliable and secure system operation. Grid voltage sags are the most concerning issues and further research has to be done on system performance under such dynamic conditions. Grid voltage sags present symmetrical positive- and negative-sequence components. If the negative sequence of the grid currents is not controlled, the power injected into the ac grid is not constant and has some ripple at twice the grid frequency. This produces dc bus voltage ripples, which may cause some critical issues to the GCPS. To solve this problem, a negative sequence current controller can be included to provide proper voltage references to the grid-connected voltage source inverter (VSI). This control should contain the ability to ride-through any types of faults by supporting the grid voltage with reactive power injection.

Different strategies to determine the references for the current control loops with different control frames have been investigated. In [2], the target is to maintain the dc-link voltage constant under unbalanced grid voltage conditions using proportional-integral (PI) controllers in the current control loops. The calculation of proper current references is addressed in [3] and a remedy to compensate for the power ripple is also introduced. In [4], a strategy to provide both active and reactive power under unbalanced voltage conditions is used. However, only the reactive power is considered in the negative-sequence current and the active power is imposed to be zero. Two different methods considering the oscillating power components produced in the filters have been studied in [5]. Finally, in [6], three different current controllers have been compared based on symmetrical components using a linear quadratic regulator.

In all the papers discussed above that are based on PI controllers in their current control loops, there is an issue of choosing proper PI controller parameters. The reason is that, in order to extract the positive and negative sequences from the grid currents, the measured magnitudes have to be filtered to remove low-frequency ripples produced during unbalanced voltage sag conditions. The filtering process produces delays and may make the system unstable.

This issue has not been yet addressed in any technical paper. This paper is focused on determining the values of the PI controller parameters that make the system stable when considering the delays caused by filtering the measured currents. These studies are done when the target is to deliver constant active power to the grid during the voltage sags. However, this is also applicable when the target is to keep the dc-link voltage constant.

The rest of the paper is organized as follows. First, the system description is defined in Section II. In Section III, the system under study is introduced. The performance of the system is assessed in two cases; when operating with a constant dc-link voltage provided by a dc source, which is discussed in Section IV, and when the VSI regulates the dc-link voltage, which is evaluated in Section V. Finally, Section VI summarizes the main conclusions of the paper. 


\section{SYSTEM DESCRIPTION AND EQUATIONS}

\section{A. Phase-Lock-Loop (PLL) Circuit}

Grid voltage synchronization is usually performed by a conventional synchronous reference frame PLL (SRF-PLL) for balanced cases. However, under unbalanced grid voltage conditions, the conventional SRF-PLL does not perform properly because the detected angle contains low frequency ripples, as proved in [7]. Therefore, a more elaborated PLL should be used capable of detecting the positive voltage sequence under unbalanced conditions. In this paper, an alternative PLL technology is used based on moving average filters (MAFs) introduced in [8] and implemented in [7].

\section{B. Current Reference Generation Process}

For a grid-connected VSI, when only the positive-sequence component is controlled, the active current reference value is obtained from the dc-link voltage and the reactive current reference is obtained through an independent method such as the well-known droop control [9]. However, when controlling both the positive- and negative-sequence components, the dc-link is no longer regulated by only the positive-sequence active current reference, but it is also affected by the positive- and negativesequence active and reactive current references. The instantaneous active and reactive power is given by

$$
\begin{aligned}
& p=P_{0}+P_{c 2} \cos (2 \omega t)+P_{s 2} \sin (2 \omega t), \\
& q=Q_{0}+Q_{c 2} \cos (2 \omega t)+Q_{s 2} \sin (2 \omega t),
\end{aligned}
$$

where $P_{0}$ and $Q_{0}$ are average values of the instantaneous active and reactive powers respectively, and $P_{c 2}, P_{s 2}, Q_{c 2}$ and $Q_{s 2}$ are second-order harmonic terms of these instantaneous powers. Expressing the relative voltage and currents in a synchronous reference frame $(d q)$ to calculate the power terms, one obtains:

$$
\begin{aligned}
& P_{0}=\left(e_{d}^{+} i_{d}^{+}+e_{q}^{+} i_{q}^{+}+e_{d}^{-} i_{d}^{-}+e_{q}^{-} i_{q}^{-}\right), \\
& P_{c 2}=\left(e_{d}^{-} i_{d}^{+}+e_{q}^{-} i_{q}^{+}+e_{d}^{+} i_{d}^{-}+e_{q}^{+} i_{q}^{-}\right), \\
& P_{s 2}=\left(e_{q}^{-} i_{d}^{+}-e_{d}^{-} i_{q}^{+}-e_{q}^{+} i_{d}^{-}+e_{d}^{+} i_{q}^{-}\right), \\
& Q_{0}=\left(e_{q}^{+} i_{d}^{+}-e_{d}^{+} i_{q}^{+}+e_{q}^{-} i_{d}^{-}-e_{d}^{-} i_{q}^{-}\right), \\
& Q_{c 2}=\left(e_{q}^{-} i_{d}^{+}-e_{d}^{-} i_{q}^{+}+e_{q}^{+} i_{d}^{-}-e_{d}^{+} i_{q}^{-}\right), \\
& Q_{s 2}=\left(-e_{d}^{-} i_{d}^{+}-e_{q}^{-} i_{q}^{+}+e_{d}^{+} i_{d}^{-}+e_{q}^{+} i_{q}^{-}\right) .
\end{aligned}
$$

It should be mentioned that (2) is based on considering the $d q$ transformation to be power conservative. These equations can be converted to a $6 \times 4$ matrix which is not reversible. The terms $P_{\mathrm{c} 2}$ and $P_{\mathrm{s} 2}$ in (2) are the ones that produce power ripples into the grid. Therefore, only the first four terms in (2) need to be controlled. The current references can be obtained as follows:

$$
\left[\begin{array}{l}
i_{d}^{+*} \\
i_{q}^{+*} \\
i_{d}^{-*} \\
i_{q}^{-*}
\end{array}\right]=\left[\begin{array}{cccc}
e_{d}^{+} & e_{q}^{+} & e_{d}^{-} & e_{q}^{-} \\
e_{d}^{-} & e_{q}^{-} & e_{d}^{+} & e_{q}^{+} \\
e_{q}^{-} & -e_{d}^{-} & -e_{q}^{+} & e_{d}^{+} \\
e_{q}^{+} & -e_{d}^{+} & e_{q}^{-} & -e_{d}^{-}
\end{array}\right]^{-1}\left[\begin{array}{l}
P_{0}^{*} \\
P_{2 c}^{*} \\
P_{2 s}{ }^{*} \\
Q_{0}{ }^{*}
\end{array}\right]
$$

In this paper, the target is to deliver constant power to the grid during the voltage sag process, therefore the terms $P_{c 2}^{*}$ and $P_{s 2}^{*}$ should be set to zero for this purpose.

\section{Voltage and Current Components Extraction}

When controlling only the positive sequence, using the angle extracted from the PLL explained in Section II- $A$, the extracted current sequence components are constant with no ripples during unbalanced voltage conditions. However, when the control target is to achieve constant power into the grid, the currents have to be unbalanced by including a negative sequence. Therefore, when the currents are measured and transformed into $d q$ frames rotating in opposite directions, both sequences interact with each other producing ripples at twice the fundamental frequency [10], i.e. $100 \mathrm{~Hz}$ when the grid frequency is $50 \mathrm{~Hz}$. As a result, a filtering technique is needed to remove these ripples. Moving average filters (MAFs) can be used for this purpose, as shown in Fig. 1. The same procedure is applied to extract the positive- and negative-sequence components from the grid voltages.

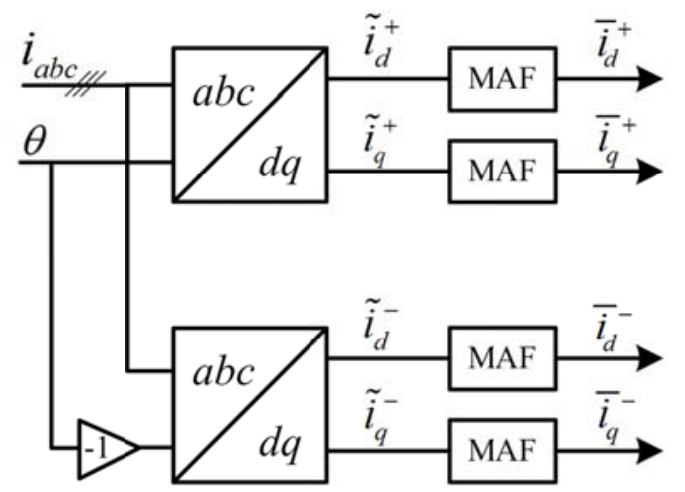

Fig. 1. Currents sequences extraction using MAFs.

The equation of the MAF is as follows:

$$
\bar{x}(t)=\frac{1}{T_{w}} \int_{t-T_{w}}^{t} x(\tau) d \tau,
$$

in which $T_{w}$ is the window width of the MAF. It is proved in [10] that under unbalanced voltage conditions a MAF with 
$T_{w}=T / 2, T$ being the grid period, can remove all the second-order harmonics. The filtering process introduces a delay, regardless of the filtering technique implemented. In the case of using MAFs, the delay is equal to the window width $T_{w}$.

\section{Current Control Loop}

The current control loop is composed of two parallel loops for positive- and negative-sequence controls. Each loop contains two PI controllers as well as decoupling terms $(\omega L)$ and feedforward terms from the $d q$ transformed grid voltages, as shown in Fig. 2. Current control loop The same system is used in parallel for the negative-sequence control. The only difference is that the sign of $\omega L$ is negative due to the inverse direction of the negative-sequence vector rotation. Finally, the summation of the positive- and negative-sequence voltage components provides the reference voltage to the inverter $\left(v_{a b c}^{*}\right)$.

As depicted in Fig. $2, \bar{i}_{d}^{+}$and $\bar{i}_{q}^{+}$are the extracted positivesequence currents (Fig. 1), $\bar{e}_{d}^{+}$and $\bar{e}_{q}^{+}$are extracted positivesequence voltages, and finally $v_{d}^{+*}$ and $v_{q}^{+*}$ are the positivesequence voltage references for the VSI. It should be mentioned that the grid voltage components used in the matrix (3) to obtain the current references are the filtered components, i.e. $\bar{e}_{d}^{+}, \bar{e}_{q}^{+}$, $\bar{e}_{d}^{-}, \bar{e}_{q}^{-}$. Therefore, these measurements are also delayed because of the MAFs when there is a voltage sag process.

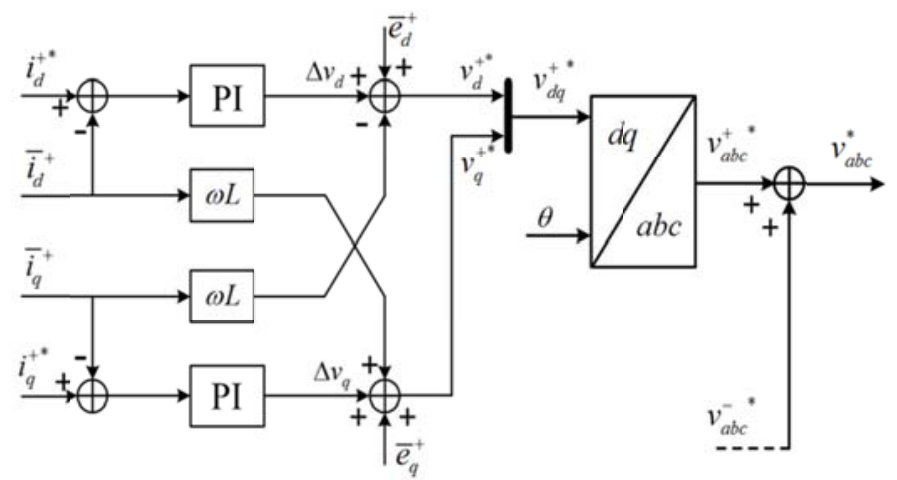

Fig. 2. Current control loop.

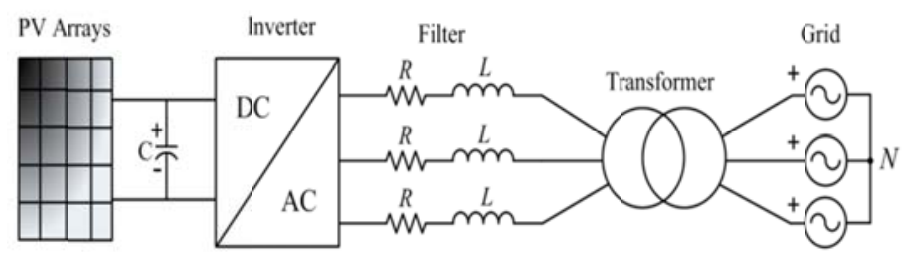

Fig. 3. Schematic of the case study system.

\section{CASE STUdy SySTEM}

The case study system is a single-stage inverter-based threephase grid connected PV system which is shown in Fig. 3. The generated power by PV array in the standard test conditions (STCs) is $1 \mathrm{MW}$, the rated ac power of the inverter is $1.1 \mathrm{MVA}$, the transformer is $1.2 \mathrm{MVA}, 20 \mathrm{kV} / 415 \mathrm{~V}$, Dny 11 and $50 \mathrm{~Hz}$, and the ac source is the representative of a stiff grid of $20 \mathrm{kV}$.

The low voltage side of the transformer $(415 \mathrm{~V})$ is connected to the inverter and the instantaneous voltage magnitudes are represented by $e_{a b c}$. Between the inverter and the transformer there are filtering inductances with $R=1 \mathrm{~m} \Omega /$ phase and $L=100 \mu \mathrm{H} /$ phase. A two-phase fault with $50 \%$ voltage drop in phase $a$ and $40 \%$ in phase $b$ is applied at the low-voltage side of the transformer for all the later tests.

\section{ANALYSis Operating With a DC-Link Voltage SOURCE}

First, the performance of the system is evaluated when the dc-link is kept constant by a dc voltage source. During the steady-state conditions the power reference $P_{0}^{*}$ is set to $1 \mathrm{MW}$ and $Q_{0}{ }^{*}$ is set to zero. However, $P_{0}{ }^{*}$ is set to zero and $Q_{0}{ }^{*}$ is set to $0.8 \mathrm{MV}$ ar during the voltage sag process to address the faultride-through (FRT) requirement.

\section{A. Stability Analysis}

Before evaluating the system performance during the voltage sags, the stability of the current control loop when applying the MAFs is analysed. The delay that the MAFs introduce to the current control loops deteriorate the fast dynamics and may lead to instability.

Converting the MAF operator (4) to the s-domain:

$$
\bar{X}(s)=\frac{1}{T_{w} s}\left[1-e^{-T_{w} s}\right] X(s) .
$$

Considering the average model of the current control loop when compensating for the coupling terms [11], two independent blocks are obtained as in Fig. 4. The same average control loops are applied to the negative-sequence current control loop.

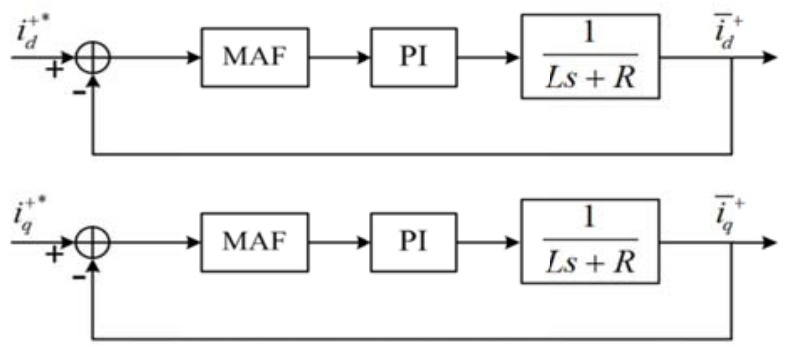

Fig. 4. Average current control loops after compensating for the coupling terms. 


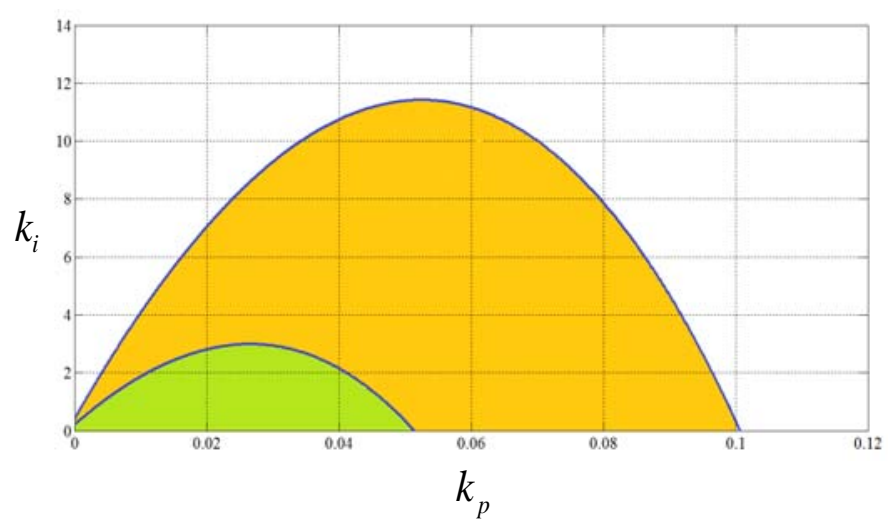

Fig. 5. Stable region for the parameters of the PI current controllers when considering the MAFs in the extraction of the positive- and negative sequences of the grid currents.

To obtain the stability region for the PI controller parameters, first, the MAF is linearized through the 'padeapproximation' method, which is available in MATLAB toolbox. After linearization, the method in [12] is selected to calculate the stable region for the PI controller parameters.

Considering the filtering parameters and a $5^{\text {th }}$ order approximation of the MAFs, the stable region for the PI controller parameters is the small green area in Fig. 5. Regarding to the other filtering methods such as the one in [6], with a delay half of the MAF used in this paper, i.e. a quarter of the grid voltage period $(T / 4)$, the stable region is considerably larger, involving the orange and green areas in Fig. 5. Therefore, as expected, smaller delays lead to have wider stable area for the PI controllers' parameters in the current control loops.

\section{B. Simulations}

The green region in Fig. 5 defines low values to the PI parameters $\left(k_{p}, k_{i}\right)$. Therefore, the dynamic performance of the controller will be slow. After several tests plotting step responses, the values adopted for the PI parameters $\left(k_{p}, k_{i}\right)$ are $(0.015,0.15)$. Using these parameters for the test system, the response during the steady-state and fault time is as shown in Fig. 6. As shown in this figure, during the voltage sag process and after fault removal, the dynamics of the PI controllers are relatively slow. Nevertheless, the system remains stable in both steady-state and fault conditions.

The use of the MAFs to filter the grid currents has a significant impact on the dynamics of the current control loop. Furthermore, the measured grid voltages are also filtered in order to be used as feed-forward terms, as shown in Fig. 2. Again this filtering process produces some delays that deteriorate the dynamic of the system. A proposed solution to improve the dynamics of the current loops is to include the grid voltages $e_{a b c}$ as feed-forward terms added after the summation of the positive and negative voltage references of the inverter. Therefore, the feed-forward voltage terms do not need to be filtered and the inverter voltage references follow the changes in the grid voltages faster. The improved currents with the same parameters of the PI controller are depicted in Fig. 7(a) and the generated active and reactive powers are shown in Fig. 7(b). For the PI parameter pairs out of the stable boundary in Fig. 5, the system becomes unstable, which is not shown here. It should be mentioned that the system in [6] has been analyzed with a constant dc source. However, for a real PV system the dc-link voltage should be regulated and this case is studied in Section V.

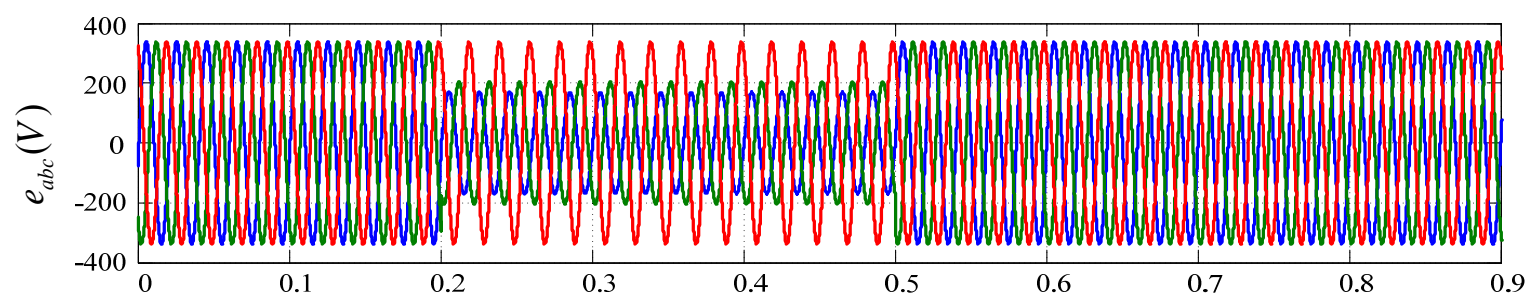

(a)

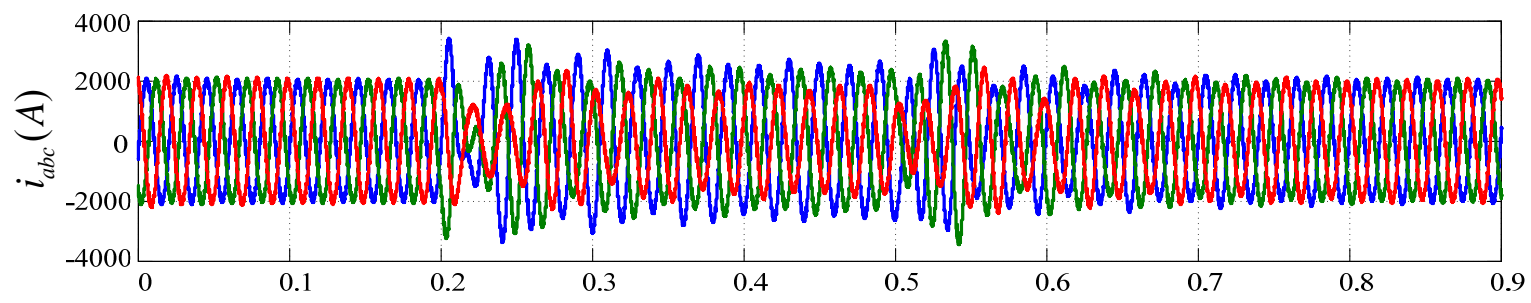

(b)

Time $(s)$

Fig. 6. (a) Voltages and (b) output currents when choosing PI parameters from the stable region and constant dc-link voltage imposed by a dc voltage source. 

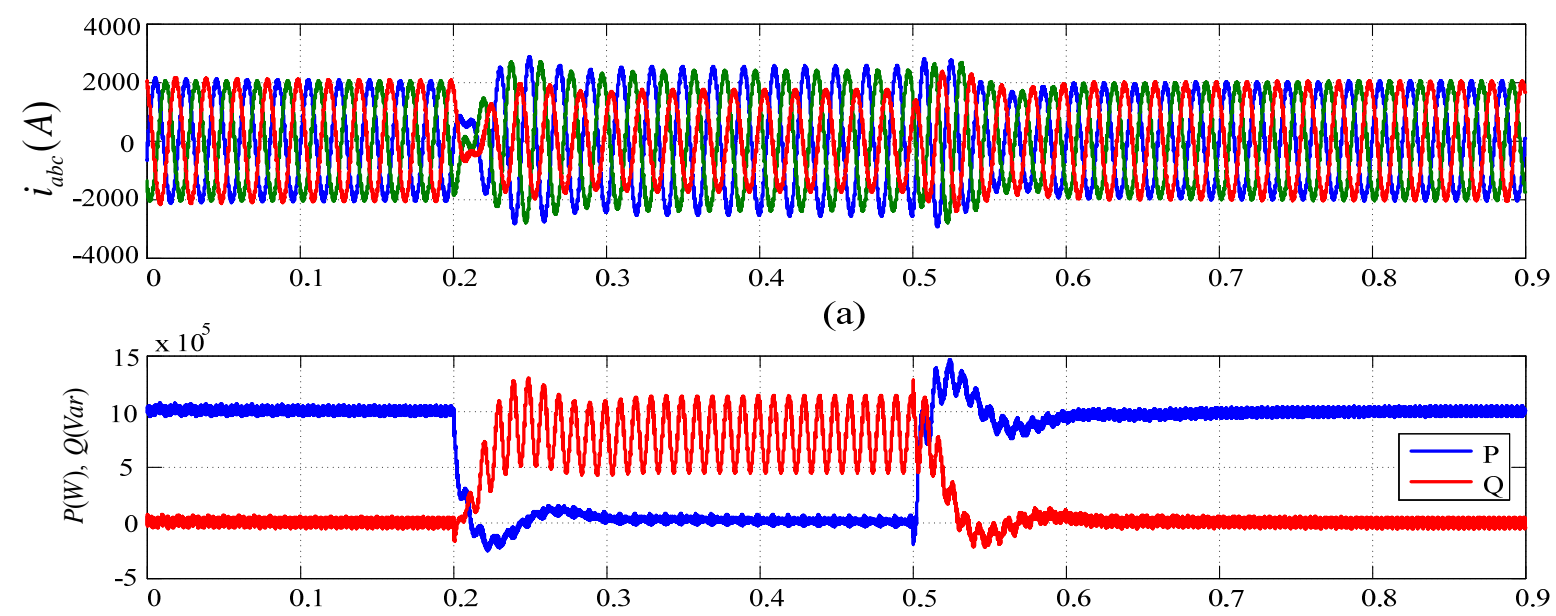

(b)

Time $(s)$

Fig. 7. (a) Improved output currents after applying the grid voltages as feed-forward terms at the end of the current control loops and (b) related active and reactive powers.

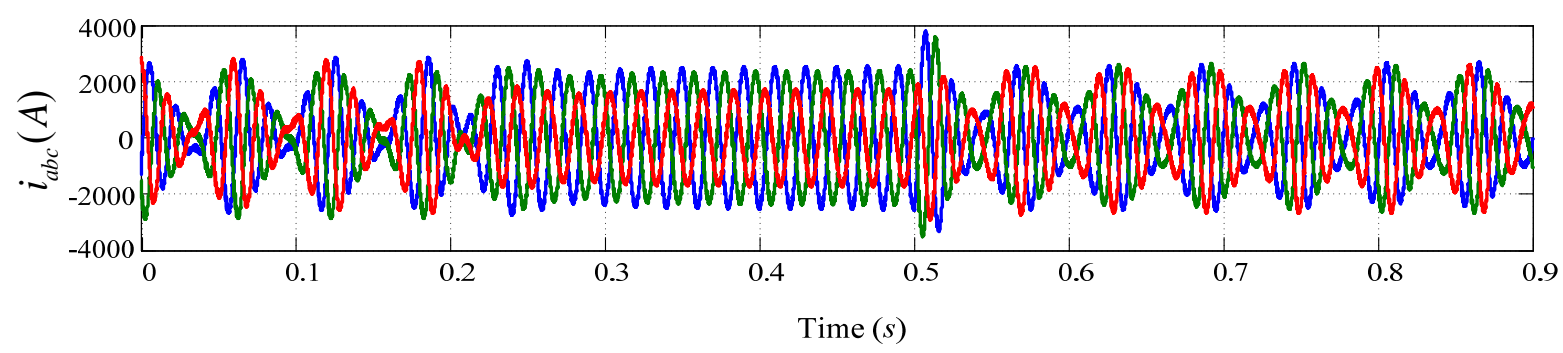

Fig. 8. Output currents when the system regulates the dc-link voltage.

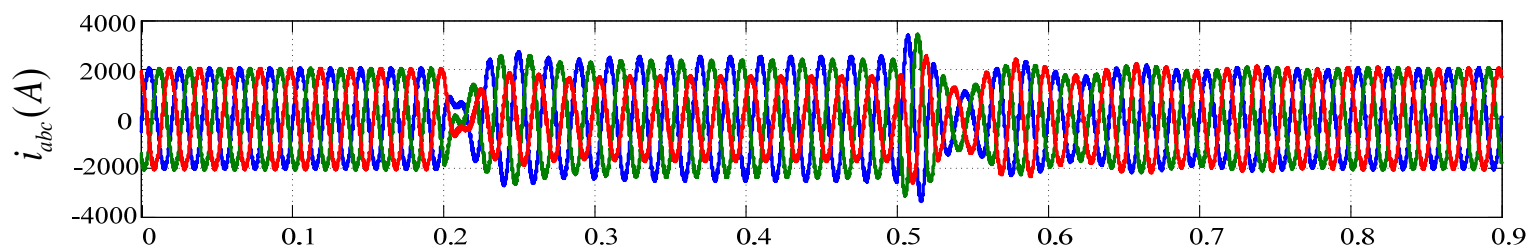

(a)

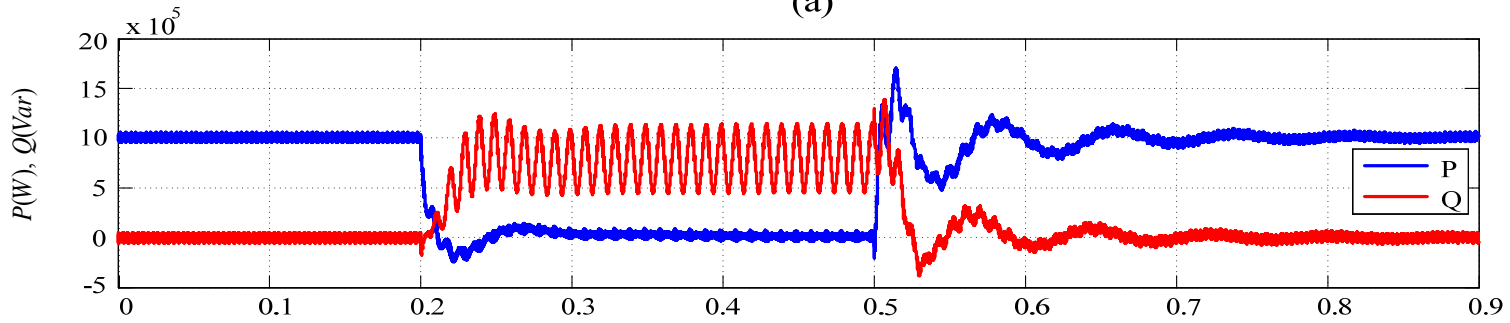

(b)

Time $(s)$

Fig. 9. Waveforms when the system regulates the dc-link voltage using low values in the PI parameters of the external loop (dc-link voltage loop). (a) Grid currents and (b) generated active and reactive powers. 


\section{ANALYSIS OPERATION WITH DC-LinK VOLTAGE REgUlation}

In this section the performance of the system when regulating the dc-link voltage is assessed. During the steadystate conditions $P_{0}^{*}$ is obtained by regulating the dc-link voltage while during the voltage sag it is set to zero. The reactive power reference is as in the previous section. Moreover, as the target is to deliver constant power to the grid during the voltage sag, there will be power fluctuations in the grid filter because of the unbalanced grid currents which will cause the dc-link voltage to oscillate. Therefore, a MAF is used to filter these ripples from the dc-link voltage measurement.

The performance of the system is assessed when there is a MAF in the measurement of the dc-link voltage control loop as well as the inner current control loops. With the same parameters as those used in the previous section, the results for the PI controllers of the current control loops with settling time $t_{\mathrm{s}}=41 \mathrm{~ms}$, and $(7.65,489)$ for the dc-link PI controller $\left(t_{\mathrm{s}}=78 \mathrm{~ms}\right)$ are shown in Fig. 8. The currents are oscillating which may lead to inverter disconnection. The reason is that the slow dynamics of the current control loops due to the MAFs interact with the external loop (dc-link voltage loop) dynamics. The dynamics of the current control loops should be at least five times faster than the dynamic of external loop to be able to analyze them separately. However, with the inclusion of the MAFs in the control loops, the dynamic of external an internal loops become almost similar.

The one alternative to improve the performance of the system is to retune the dynamics of the external loop to be slower; however, it will affect the dynamics of the whole system which is not actually desirable. The new parameters chosen for the PI controller of the voltage loop is $(2.16,39)$, which produce the settling time of $t_{s}=176 \mathrm{~ms}$. The grid currents are depicted in Fig. 9(a) and the generated active and reactive powers are shown in Fig. 9(b). The system is stable; however, the dynamics are relatively slow.

\section{CONCLUSION}

In this paper, the performance of a GCPS under unbalanced voltage conditions considering both positive and negative sequences is studied. Using PI controllers for the current loops, implementation of a filtering technique is necessary to extract the $d q$ components of the voltages and currents. However, all the filtering techniques introduce some delays and slow down the dynamics of the controllers. As a consequence, these filtering techniques restrict the stable region of the parameters of the PI controllers. Furthermore, low values for the PI parameters have to be chosen.
In addition, if the dc-link voltage is regulated by an external loop, the dynamics of that controller need to be relatively slow compared to the current control loops in order to achieve stable operation of the system. Therefore, the overall dynamic performance is deteriorated considerably. An alternative to the use of PI controllers would be using proportional-resonant controllers. Since the positive and negative sequences of the grid currents would not be needed in the control loops, faster dynamics are expected to be achieved. Therefore, our future research will be focused on the use of PR controllers.

\section{REFERENCES}

[1] F. Katiraei and J. R. Aguero, "Solar PV integration challenges," IEEE Power Energy Mag., vol. 9, pp. 62-71, Apr. 2011.

[2] Yin Bo, R. Oruganti, S. K. Panda, and A. K. S. Bhat, "An output-powercontrol strategy for a three-phase PWM rectifier under unbalanced supply conditions," IEEE Trans. Ind. Electron., vol. 55, pp. 2140-2151, May 2008.

[3] A. Junyent-Ferre, O. Gomis-Bellmunt, T. C. Green, and D. E. SotoSanchez, "Current control reference calculation issues for the operation of renewable source grid interface VSCs under unbalanced voltage sags," IEEE Trans. Power Electron., vol. 26, pp. 3744-3753, Dec. 2011.

[4] Lee Chia-Tse, Hsu Che-Wei, and Cheng Po-Tai, "A low-voltage ridethrough technique for grid-connected converters of distributed energy resources," IEEE Trans. Ind. Appl., vol. 47, pp. 1821-1832, Jul. 2011.

[5] F. A. Magueed, A. Sannino, and J. Svensson, "Transient performance of voltage source converter under unbalanced voltage dips," in Proc. IEEE Annual Power Electronics Specialists Conference (PESC), 2004, pp. 1163 1168 .

[6] S. Alepuz, S. Busquets-Monge, J. Bordonau, J. A. Martinez-Velasco, C. A Silva, J. Pontt, and J. Rodriguez, "Control strategies based on symmetrical components for grid-connected converters under voltage dips," IEEE Trans. Ind. Electron., vol. 56, pp. 2162-2173, Jun. 2009.

[7] M. Mirhosseini and V. G. Agelidis, "Performance of large-scale gridconnected photovoltaic system under various fault conditions," in Proc. IEEE International Conference on Industrial Technology (ICIT), 2013, pp. 1775-1780.

[8] E. Robles, S. Ceballos, J. Pou, J. L. Martin, J. Zaragoza, and P. Ibanez, "Variable-frequency grid-sequence detector based on a quasi-ideal lowpass filter stage and a phase-locked loop," IEEE Trans. Power Electron., vol. 25 , pp. 2552-2563, Oct. 2010.

[9] E. Troester, "New german grid codes for connecting PV systems to the medium voltage power grid," presented at the International Conference on Concentrating Photovoltaic Power Plant, Germany, 2009.

[10] M. Mirhosseini, J. Pou, and V. G. Agelidis, "Current improvement of a grid-connected photovoltaic system under unbalanced voltage conditions," in Proc. IEEE Annual International Energy Conversion Congress and Exhibition (ECCE ASIA), Melbourne, Australia, 2013, pp. 66-72.

[11] E. Robles, S. Ceballos, J. Pou, F. Salcedo, and M. Santos, "Tuning of controllers in grid-connected multilevel converters," International Review on Modelling and Simulations (IREMOS), vol. 5, pp. 1165-1174, Jun. 2012.

[12] N. Tan, I. Kaya, and D. P. Atherton, "Computation of stabilizing PI and PID controllers," in Proc. IEEE Conference on Control Applications (CCA), 2003, pp. 876-881. 